\title{
A Feature Set Decomposition Method for the Construction of Multi-classifier Systems Trained with High-Dimensional Data
}

\author{
Yoisel Campos ${ }^{1}$, Roberto Estrada ${ }^{1}$, Carlos Morell ${ }^{2}$, and Francesc J. Ferri ${ }^{3}$ \\ 1 Univ. de Holguín "Oscar Lucero Moya". Holguín, Cuba \\ campos.yoisel@gmail.com, restradal@facinf.uho.edu.cu \\ 2 Computer Science Dept. Univ. Central \\ "Marta Abreu" de Las Villas. Santa Clara, Cuba \\ cmorellp@uclv.edu.cu \\ 3 Dept. d'Informàtica Universitat de València València, Spain \\ francesc.ferri@uv.es
}

\begin{abstract}
Data mining for the discovery of novel, useful patterns, encounters obstacles when dealing with high-dimensional datasets, which have been documented as the "curse" of dimensionality. A strategy to deal with this issue is the decomposition of the input feature set to build a multi-classifier system. Standalone decomposition methods are rare and generally based on random selection. We propose a decomposition method which uses information theory tools to arrange input features into uncorrelated and relevant subsets. Experimental results show how this approach significantly outperforms three baseline decomposition methods, in terms of classification accuracy.
\end{abstract}

Keywords: multi-classifier systems, feature set decomposition, information theory.

\section{Introduction}

The technological advancement on the field of sensors and other measuring methodologies, has provoked the existence of processes which output data described by a large amount of features. These are often termed high-dimensional datasets and their distinctive quality is that the number $m$ of features describing the data is far greater than the number $N$ of data instances $(m \gg N)$.

Performing machine learning from such datasets is of high interest. Not only because of the inherent usefullness of discovering the underlying patterns, but also because frequently there is poor backgorund knowledge about the domains from where the data are drawn. Certain domains, e.g. genetic studies or biochemistry, yield datasets described by a number of features in the order of $10^{4}$, while containing only a few hundred instances [14. This extreme disproportion conducts to the appearance of certain fenomena affecting the performance of most machine learning algorithms, which is known in the literature as the "curse" of dimensionality [6].

J. Ruiz-Shulcloper and G. Sanniti di Baja (Eds.): CIARP 2013, Part I, LNCS 8258, pp. 278-285, 2013.

(C) Springer-Verlag Berlin Heidelberg 2013 
Many strategies have been applied to tackle this issue. One of them is the construction of multi-classifier systems (MCS) [2, where several complementary models are combined in some way to make predictions. For high-dimensional problems, MCS generally train each individual classifier using only a subset of the original data features, with the intention of reducing the effects of dimensionality.

Such an approach is the Random Subspace Method (RSM) for constructing decision forests [8, which introduced a general framework to deal with highdimensional data. RSM iteratively selects random subsets of features from the original dataset (i.e. subspaces), which are then employed to train decision trees. This operation results in an ensemble of models trained with different projections of the data, whith the random subspacing ensuring diversity. This action of generating several subsets from an original feature set is commonly defined as "decomposition" [10.

However, there's no evidence to assert whether random decomposition is the most effective in terms of overall classification accuracy. Ideally, all features should be allocated inside a decomposition in a way that maximizes their combined usefullness. Some features will work well together, some will not because of redundancy or irrelevance.

In this paper, we explore the application of information theory tools to the decomposition task. After covering some fundamental concepts about feature set decomposition in Section 2, we present an information theoretic decomposer in Section 3, which is the main contribution of this work. Section 4 summarizes an experimental study where statistical tests show how our approach significantly outperforms 3 baseline methods, in terms of classification accuracy, over 19 highdimensonal datasets.

\section{Feature Set Decomposition for the Creation of Multi-classifiers}

Decomposition methods are rarely found as an independent field of study. Usually a non-random decomposition strategy is developed as a secondary product of a certain ensemble construction method [119]. A tight integration between the different layers of a multi-classifier is of course desirable. But standalone decomposition methods [4] present their own advantages. According to Maimon and Rokach [10], some of these advantages are:

- Increase in classification performance, due to the simplification of the task.

- Scalabiliy to high-dimensional datasets.

- Flexibility in learning algorithm selection.

Given an inducer $I$, a combination method $C$ and a training set $S$ with input feature set $F=\left\{f_{1}, f_{2}, \ldots, f_{m}\right\}$ and a target feature $c$, the goal is to find an optimal decomposition $D_{\text {opt }}$ of the input feature set $F$ into $n$ mutually exclusive subsets $D_{i}=\left\{d_{i 1}, d_{i 2}, \ldots, d_{i k}\right\} \mid d_{i j} \in F ; i=1, \ldots, n$ that are not necessarily exhaustive, such that the generalization error of the induced classifiers, combined using method $C$, will be minimized. 


\section{Information Theoretic Decomposition}

Several machine learning techniques have been inspired on information theory concepts, especially in the field of feature selection. Some works have taken advantage of the usefulness of such concepts, to measure how much information a given description feature carries about the target feature. The root concept in this domain is the entropy of a feature $X, H(X)$. The entropy of a feature could be interpreted as the amount of uncertainty in drawing, at random, a value from it [12]. Furthermore, the conditional entropy between two given features $H(X \mid Y)$ expresses how much uncertainty remains about $X$, once $Y$ is known. These two concepts are further combined to form other relational information measures, which prove to be very useful in assessing the interaction of features in a machine learning environment.

In particular, the Symmetrical Uncertainty (SU) and the Conditional Mutual Information (CMI) display some desirable properties. The success of applying these for attribute selection problems has been demonstrated in algorithms like FCBF[15] and CMIM[5].

$\mathrm{SU}$ describes how much information is shared by two given features. It is a normalized measure, with 1 indicating that the values in either feature can be predicted from the other and 0 indicating that $X$ and $Y$ are independent.

$$
S U(X, Y)=2\left[\frac{H(X)-H(X \mid Y)}{H(X)+H(Y)}\right]
$$

CMI is an estimate of the amount of information shared between $X$ and $Y$ when $Z$ is known. As shown in Equation 2, when the amount of information about $X$ contained in $Y$ is already given by $Z$, CMI equals 0 even if both $Z$ and $Y$ are informative and mutually independent.

$$
C M I(X, Y \mid Z)=H(X \mid Z)-H(X \mid Y, Z)
$$

Regardless of the success in the application of the information theory approach in attribute selection algorithms, the selection problem essentially differs from the decomposition problem. Our goal will be to simultaneously construct the desired amount of feature subsets, so that the most appropriate subset will be determined for each feature analyzed.

For this information theoretic decomposition we will traverse 3 phases, each one aiming at a key aspect of multi-classifiers construction: relevance, diversity and non-redundancy. The whole procedure is summarized in Algorithm 1

\subsection{Phase A: Relevance}

It is desirable that only informative features should be considered to form the decomposition. Hence, the first phase will ensure that non-informative features are excluded. To achieve this, all description features that show information independence from the target feature or class, will be removed.

Phase A will output a depurated feature set $F^{\prime}$, with $S U(f, c)>0 \mid \forall f \in F^{\prime}$. 


\subsection{Phase B: Diversity}

It has been proved that individual classifiers with performance error under 0.5 and weakly correlated decisions, will display a higher performance when combined in an ensemble 2]. This weak correlation among the individual decisions is usually termed diversity and represents a key property on every ensemble.

Our depurated feature set $F^{\prime}$ will most likely contain some subset of highly relevant features which are also highly independent with regard to each other. Phase B will find these features and will designate them as "seeds", from which the decomposition subsets will grow. This is accomplished through an iterative procedure, where CMI is used to determine the usefulness and diversity of features. Starting from the most informative feature, at each iteration a new feature is selected, based on the amount of additional information it provides. It becomes a seed since it carries information about the class that is not already provided by the previously selected seeds.

Phase B outputs the features that will be used as seeds to build up the decomposition subsets. Their main property is that each one of them provides an amount of information about the class that complements the others. Our intuition is that such property should enhance the ensemble diversity, once the respective feature subsets are generated from uncorrelated seeds.

\subsection{Phase C: Non-redundancy}

As a final requirement, the decomposition must decide whether to include each description feature and where to allocate it. The main goal in this phase is to distribute features among subsets, in a way that optimizes their combined usefulness to predict the class. To achieve this, every feature should be allocated to the subset where it provides most information, i.e. where it is least redundant. Furthermore, a feature that is redundant in all subsets should not be considered.

We will define the amount of redundancy that a feature $X$ introduces to a subset $Z$, as the maximum difference between the correlation of $X$ to the members of $Z$ and the relevance of $X$, as stated in Equation 3 .

$$
R(X, Z)=\max _{\forall z \in Z}[S U(X, z)-S U(X, c)]
$$

Subsequently, a feature should be allocated to the subset where it introduces the least redundancy. To further increase the flexibility of the model, a threshold could be used to tolerate certain amount of redundancy. As a result, all target features are evaluated and either discarded or included in the most appropriate subset. This phase outputs the final decomposition $D$, which can then be applied to the original dataset to train an ensemble of $n$ classifiers.

\section{Experimental Study}

In order to evaluate the performance of our information theoretic decomposer, we have tested this approach in an experimental setup. The objective is to determine 


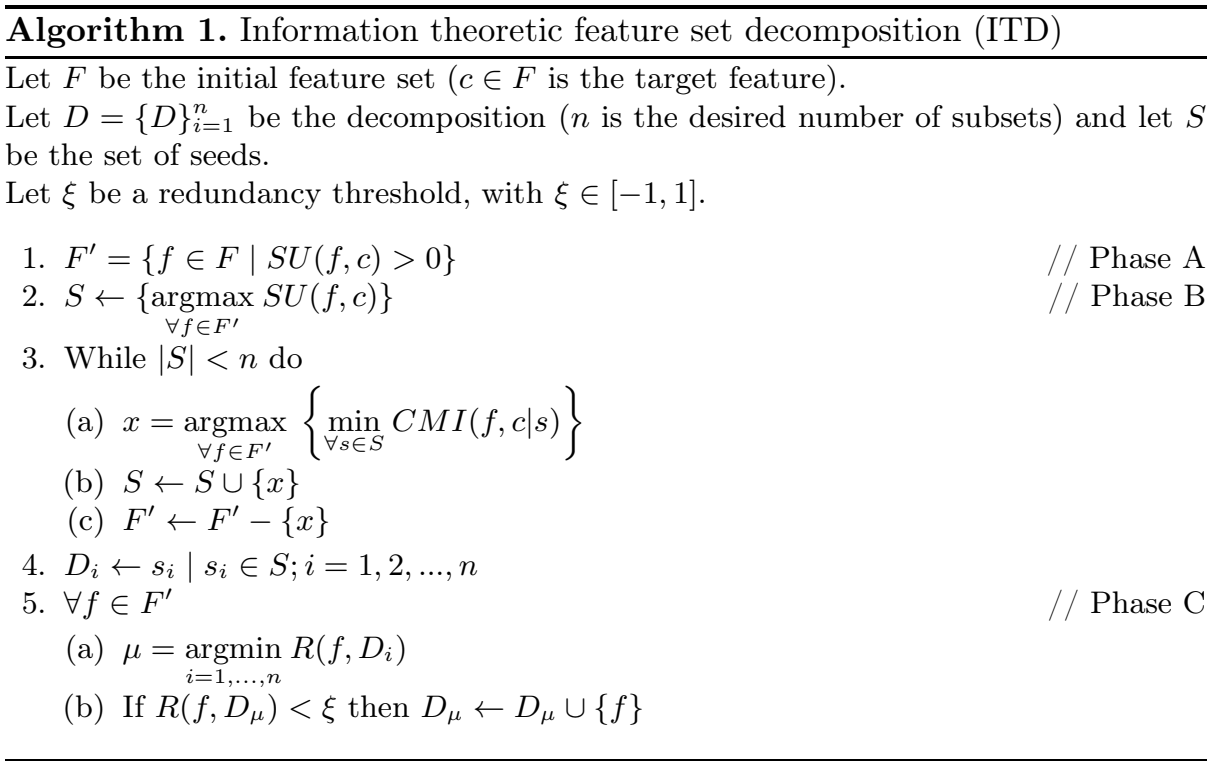

how this method compares to other baseline decomposition methods in terms of classification accuracy, when working with high-dimensional datasets.

\subsection{Datasets}

There is a number of benchmark repositories on the web providing structured high-dimensional datasets. The experimental data for this study was composed from different sources.

The NIPS 2003 feature selection challenge [7] provided us with 3 datasets: "Arcene", "Dorothea" and "Gisette". The remaining 3 datasets presented for that challenge do not comply with the desired property of $m \gg N$. Furthermore, all 6 datasets from the basic track of the RSCTC 2010 challenge on mining DNA data for medical diagnosis [14] were included in our study. In addition, a group of 10 datasets from the Kent Ridge (KR) Bio-Medical Data Set Repository 11 were also included.

A preprocessing was needed in 3 of the KR datasets (DLBCL-NIH, DLBCLStanford and Prostate Tumor), where missing values were replaced by the perclass average of the corresponding feature values, although other more suited strategies will be tested in the future. Some interesting properties of the experimental data (Table 1) are: the variety in the number of classes (ranging from 2 to 7 ), the presence of unbalanced classes (in 13 out of the 19 datasets) and the variety in the number of features and instances.

\footnotetext{
${ }^{1}$ http://datam.i2r.astar.edu.sg/datasets/krbd/
} 


\subsection{Algorithms and Methods}

The general procedure was to decompose the feature set in different ways to train a decision forest. The ensemble decision is then obtained through a standard average combination of the class posterior probabilities. Each decision tree was built using the $\mathrm{C} 4.5$ algorithm. For every decomposition method several values for the number of subsets were considered and tested: up to 50 at intervals of 5 .

Table 1. Description of the experimental data $(N$ is the number of instances, $m$ denotes the number of features) and averaged accuracy rates of the methods. The best performances for each dataset are emphasized in bold. The threshold value $(\xi)$ and number of subsets $(s)$ corresponding to de best performances of ITD are also shown.

\begin{tabular}{lcc|cccc|cc}
\hline Dataset & $N$ & $m$ & \multicolumn{7}{l|}{ RSM } & IFCBF CERP ITD & $s$ & $\xi$ \\
\hline Arcene & 200 & 10000 & 0.74 & 0.77 & $\mathbf{0 . 7 8}$ & $\mathbf{0 . 7 8}$ & 30 & 0.2 \\
Dorothea & 350 & 100000 & $\mathbf{0 . 7 6}$ & 0.65 & 0.58 & 0.71 & 5 & 0.1 \\
Gisette & 1000 & 5000 & $\mathbf{0 . 9 3}$ & 0.92 & $\mathbf{0 . 9 3}$ & $\mathbf{0 . 9 3}$ & 10 & 0.5 \\
E-GEOD-10334 & 123 & 54676 & 0.83 & $\mathbf{0 . 8 5}$ & 0.83 & 0.84 & 25 & 0.1 \\
E-GEOD-5406 & 105 & 22284 & $\mathbf{0 . 6 5}$ & $\mathbf{0 . 6 5}$ & 0.63 & $\mathbf{0 . 6 5}$ & 15 & 0.1 \\
E-GEOD-13425 & 95 & 22278 & 0.89 & 0.89 & 0.88 & $\mathbf{0 . 9 0}$ & 30 & 0.3 \\
E-GEOD-13904 & 113 & 54676 & 0.40 & 0.41 & 0.40 & $\mathbf{0 . 4 3}$ & 20 & 0.3 \\
E-GEOD-4290 & 89 & 54676 & 0.62 & 0.63 & 0.63 & $\mathbf{0 . 6 4}$ & 30 & 0.5 \\
E-GEOD-9635 & 92 & 59005 & 0.52 & 0.53 & 0.55 & $\mathbf{0 . 5 7}$ & 25 & 0.5 \\
ALL-AML Leukemia & 72 & 7130 & 0.93 & 0.92 & 0.93 & $\mathbf{0 . 9 6}$ & 20 & 0.3 \\
Breast Cancer & 97 & 24482 & 0.64 & 0.67 & $\mathbf{0 . 6 9}$ & $\mathbf{0 . 6 9}$ & 30 & 0.1 \\
Central Nervous System & 60 & 7130 & 0.51 & 0.51 & 0.53 & $\mathbf{0 . 5 5}$ & 10 & 0.1 \\
Colon Tumor & 62 & 2001 & 0.75 & 0.75 & $\mathbf{0 . 8 0}$ & 0.79 & 20 & 0.1 \\
DLBCL-NIH & 240 & 7400 & 0.53 & 0.54 & $\mathbf{0 . 5 7}$ & 0.55 & 10 & 0.3 \\
DLBCL-Stanford & 47 & 4027 & 0.87 & 0.91 & $\mathbf{0 . 9 3}$ & 0.92 & 30 & 0.1 \\
MLL Leukemia & 72 & 12583 & 0.93 & 0.95 & $\mathbf{0 . 9 6}$ & $\mathbf{0 . 9 6}$ & 30 & 0.3 \\
Lung cancer & 181 & 12534 & $\mathbf{0 . 9 8}$ & 0.97 & $\mathbf{0 . 9 8}$ & $\mathbf{0 . 9 8}$ & 10 & 0.3 \\
Prostate Tumor & 136 & 12601 & 0.87 & 0.87 & 0.89 & $\mathbf{0 . 9 0}$ & 15 & 0.2 \\
Stjude Leukemia & 327 & 12559 & 0.76 & 0.79 & 0.76 & $\mathbf{0 . 8 1}$ & 10 & 0.1 \\
\hline
\end{tabular}

We compared 4 decomposers:

- RSM, the Random Subspace Method [8], which builds subsets of randomly selected features and allows overlapping, i.e. a feature may appear in more than one subset. Three subspace sizes were tested, as proportions from the total: $0.1,0.3$ and 0.5 .

- IFBCF, an iterative application of an attribute selection algorithm, in this case FCBF [15. Each iteration builds a subset and the selected features are excluded from subsequent iterations. This particular algorithm was selected for its time efficiency and its similarity with our decomposer.

- CERP, Classiffication by Ensembles from Random Partitions [3], which is a successful decomposition method adapted from RSM. As its name states, this method randomly partitions the feature space, thus avoiding overlaps. 
- ITD, our information theoretic decomposer, which also avoids feature overlaps. Redundancy threshold values we considered were: $0.1,0.2,0.3$ and 0.5.

All implementations and tests were performed using Weka 13 3.7.6. For the experimental scheme and statistical considerations, we followed what is recommended in a work from Demšar [1]. Five repetitions of a two-fold cross-validation $(5 \times 2 \mathrm{cv})$ were performed for every combination of parameter values, with each algorithm over each dataset. The performance criterion was the balanced accuracy rate, recommended for experiments where unbalanced datasets are present [7. A Friedman test was applied to determine whether significant differences in performance exist within the group of combination algorithms. Afterwards, the the Holm procedure was used as a post-hoc test, to help identifying those significant differences. All tests were performed with a $95 \%$ confidence level.

\subsection{Experimental Results}

The performance of each method over each dataset was computed averaging the 10 performance measurements resulting from the $5 \mathrm{x} 2 \mathrm{cv}$. The best performance of each method over each dataset was selected from the different parameter settings. As a result, ITD was ranked first in 14 datasets out of 19 (Table 1).

A Friedman test was performed, it yielded significant differences on the accuracies of the 4 methods $\left(p=2.7408 \cdot 10^{-4}\right)$. The Holm procedure rejected all 3 hypotheses that claim non-significant differences between ITD and the other methods (Table 2). Hence, ITD significantly outperforms the others.

Table 2. Average rankings of the algorithms according to the Friedman test and Holm procedure table for $\alpha=0.05$

\begin{tabular}{ccccc}
\hline Algorithm Ranking & $p$ & Adjusted $p$ & \\
\hline ITD & 1.50 & - & - & - \\
CERP & 2.37 & 0.03814 & 0.05 & rejected \\
IFCBF & 2.92 & 0.00069 & 0.025 & rejected \\
RSM & 3.21 & $4.43 \cdot 10^{-5}$ & 0.0167 & rejected \\
\hline
\end{tabular}

\section{Conclusions and Future Work}

Information theory tools may provide useful insight about the internal properties of a dataset. They reveal feature interactions that are present regardless of the machine learning algorithm or training procedure. This has been sucessfully exploited in the past on the field of attribute selection, accounting for fast and effective algorithms. The same logic can be applied to decompose the feature set in a way not so different from a filter algorithm for attribute selection.

The experimental and statisical results suggest that this intuition may lead to significantly superior performances. A decomposition method that takes into account the internal properties of the dataset will provide independence from the learning algorithm, enabling its use on different scenarios. In particular, 
this may prove useful when dealing with high-dimensional datasets, since these are often unmanageable in full size, as well as difficult to pre-process because of the unavailability of background knowledge on the domain.

We think there is potential behind this result. As a next step it is essential to test this decomposition method using other learning algorithms to train the ensemble members. Furthermore, a thorough study on the diversity of such generated decompositions will most likely reveal new interesting insights. Additionally, it is relevant to adapt the current approach so that it allows overlappings on the feature subsets, thus enabling useful features to be present in more than one subset, as long as it does not affect the overall diversity.

\section{References}

1. Demšar, J.: Statistical comparisons of classifiers over multiple data sets. Journal of Machine Learning Research 7, 1-30 (2006)

2. Dietterich, T.G.: Ensemble methods in machine learning. In: Kittler, J., Roli, F. (eds.) MCS 2000. LNCS, vol. 1857, pp. 1-15. Springer, Heidelberg (2000)

3. Ahn, H., et al.: Classiffication by ensembles from random partitions of highdimensional data. Computational Statistics \& Data Analysis 51, 6166-6179 (2007)

4. Hu, Q., et al.: Ensemble rough subspaces. Pattern Recognition 40, 3728-3739 (2007)

5. Fleuret, F.: Fast binary feature selection with conditional mutual information. Journal of Machine Learning Research 5, 1531-1555 (2004)

6. François, D.: High-dimensional data analysis: optimal metrics and feature selection. PhD thesis, Université Catholique de Louvain (2007)

7. Guyon, I., Gunn, S.R., Ben-Hur, A., Dror, G.: Design and analysis of the NIPS 2003 challenge. In: Guyon, I., Nikravesh, M., Gunn, S., Zadeh, L.A. (eds.) Feature Extraction. STUDFUZZ, vol. 207, pp. 237-263. Springer, Heidelberg (2006)

8. Ho, T.K.: The random subspace method for constructing decision forests. IEEE Transactions on Pattern Analysis and Machine Intelligence 20, 832-844 (1998)

9. Liao, Y., Moody, J.: Constructing heterogeneous committees via input feature grouping. In: Advances in Neural Information Processing Systems, vol. 12, pp. 921-927 (2000)

10. Maimon, O., Rokach, L.: Decomposition methodology for knowledge discovery and data mining. World Scientific (2005)

11. Tahir, M.A., Smith, J.: Creating diverse nearest-neighbour ensembles using simultaneous metaheuristic feature selection. Pattern Recognition Letters 31(11), 1470-1480 (2010)

12. Torkkola, K.: Information-Theoretic Methods. In: Guyon, I., Nikravesh, M., Gunn, S., Zadeh, L.A. (eds.) Feature Extraction. STUDFUZZ, vol. 207, pp. 167-185. Springer, Heidelberg (2009)

13. Witten, I.H., Frank, E.: Data Mining: Practical Machine Learning Tools and Techniques, 3rd edn. Morgan Kaufmann Publishers (2011)

14. Wojnarski, M., et al.: RSCTC'2010 discovery challenge: Mining DNA microarray data for medical diagnosis and treatment. In: Szczuka, M., Kryszkiewicz, M., Ramanna, S., Jensen, R., Hu, Q. (eds.) RSCTC 2010. LNCS (LNAI), vol. 6086, pp. 4-19. Springer, Heidelberg (2010)

15. Yu, L., Liu, H.: Feature selection for high-dimensional data: A fast correlationbased filter solution. In: Machine Learning, ICML 2003, Washington, DC, USA, August 21-24, pp. 856-863 (2003) 